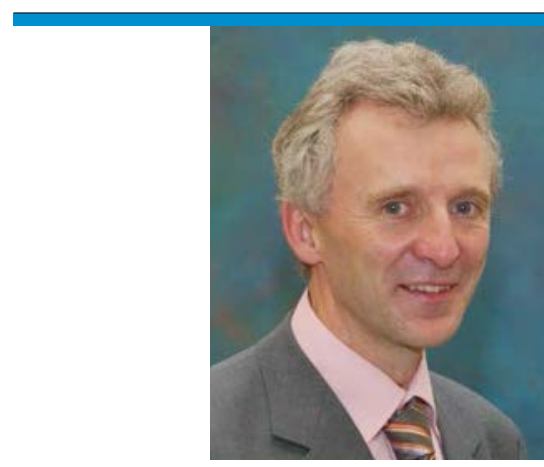

\section{Vorlesen fördert die Hirnentwicklung}

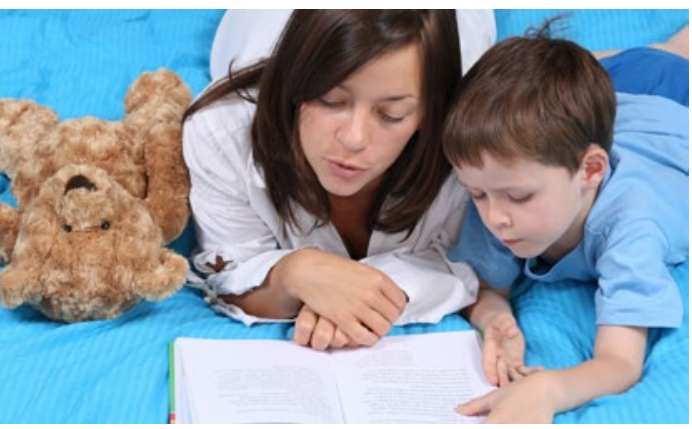

Das Vorlesen von Büchern durch die Eltern oder Großeltern gehört für Kinder zu ganz wichtigen Türöffnern für das Lesen und Schreiben. Eine interessante amerikanische Studie hat nun bei 3- bis 5-jährigen Kindern longitudinal untersucht, ob das regelmäßige Vorlesen Auswirkungen auf die Aktivierung bestimmter Hirnareale im funktionalen MRT hat [Hutton JS et al. Pediatrics 2015;136:466-78]. Die Ergebnisse zeigten, dass die Kinder, denen häufiger zuhause vorgelesen wurde, eine stärkere Aktivierung des linksseitigen parieto-temporooccipitalen Assoziationskortex hatten. Die Ergebnisse waren stabil, auch nach Korrektur auf das Haushaltseinkommen. Dies ist eine - wie ich finde - wunderschöne Bestätigung dafür, wie wichtig das Vorlesen in den ersten Lebensjahren ist.

\section{Hilft Händewaschen gegen Grippe?}

Ob einfache Maßnahmen wie das Händewaschen einen präventiven Effekt auf Atemwegsinfekte haben kann, wurde in England über 3 Jahre hinweg untersucht. 20.266 Erwachsene wurden entweder einem internetbasierten Schulungsprogramm über regelmäßiges Händewaschen inklusive Motivation und Feedback oder der Kontrollgruppe ohne Intervention zugeteilt

\title{
Fundstücke
}

\section{Dr. Martin Claßen, Chefredakteur}

[Little P et al. Lancet. 2015; pii: S01406736(15)60127-1]. In der Folge wurde nach der Zahl der Atemwegsinfekte gefragt. Nach 16 Wochen berichteten $51 \%$ der Interventionsgruppe über eine oder mehrere Episoden eines Luftwegsinfektes, gegenüber $59 \%$ in der Kontrollgruppe (Odds Ratio: $0,86, p<0,0001)$. Darüber hinaus reduzierte die Intervention die Übertragung von Infekten von Haushaltsmitgliedern auf die Probanden und umgekehrt. Dies zeigt, dass wir unseren Patienten und deren Familien gerade in der Infektsaison diese einfache Maßnahme immer wieder empfehlen sollten. Allerdings bleibt die Rate von Infekten trotzdem erstaunlich hoch.

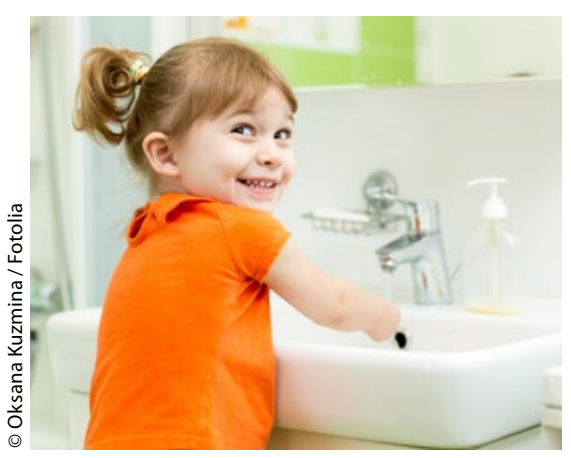

\section{Vitamin D und Nahrungsallergierisiko}

Studien zur Beeinflussung des Allergierisikos durch Vitamin D und Sonnenexposition in der Schwangerschaft und auch bei Säuglingen haben kein einheitliches Bild ergeben. Nun wurde untersucht, welchen Einfluss der Vitaminspiegel im Zusammenspiel mit dem Vitamin-D-Bindungsprotein auf die Manifestation von Nahrungsmittelallergien hat. In der populationsbasierten HealthNuts-Studie aus Australien wurden 5.276 Kinder prospektiv untersucht [Koplin JJ et al. J Allergy Clin Immunol 2015 Aug 7]. Kinder mit erniedrigtem Vitamin-DSpiegel bei der Geburt hatten nach einem Jahr gegenüber der Kontrollgruppe ein

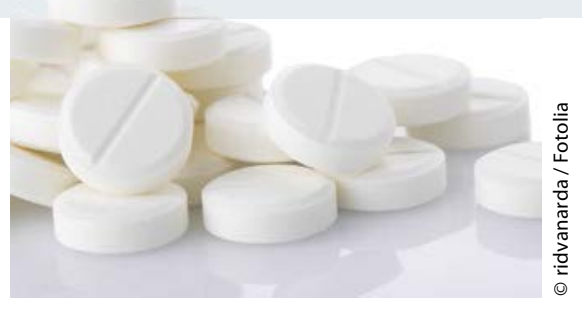

deutlich erhöhtes Allergierisiko (Odds Ratio: 6,0). Dies galt aber nur, wenn eine hohe Menge an Vitamin-D-Bindungsprotein und weniger freies Vitamin D vorhanden war. Bei niedrigem Bindungsprotein hatte ein erniedrigter Vitamin-D-Spiegel keine nachteiligen Folgen. Es steht zu erwarten, dass die weitere Analyse des Bindungsproteins einige andere offene Fragen zur Bedeutung von Vitamin D klären werden. Bis dahin würde ich zur Substitution bereits in der Schwangerschaft raten.

\section{Frühreife Mädchen trinken häufiger}

Je früher bei Jugendlichen der Alkoholabusus beginnt, umso höher ist das Risiko von Rauschtrinken und Alkoholabhängigkeit. Die Hypothese, dass eine frühe pubertäre Entwicklung bei Mädchen einen Einfluss auf den Beginn des Alkoholkonsums hat, wurde von einer Gruppe von Wissenschaftlern in Schweden an 957 Mädchen zwischen 12 und 14 Jahren aus weiterführenden Schulen belegt [Dickson DJ et al. Pediatrics 2015;136:61724]. Die früh gereiften Jugendlichen hatten ein 1-3fach höheres Risiko einer Alkoholintoxikation, insbesondere dann, wenn sie wenig elterliche Aufsicht erfuhren. Es wird

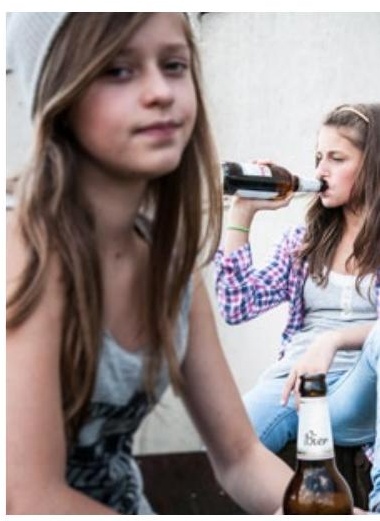
vermutet, dass diese Gruppe sich eher älte- $\frac{-\underline{\underline{0}}}{}$ ren (und damit alkoholtrinkenden) Jugendlichen anschließt, weil sie in ihrer Altersgruppe aufgrund der frühen biologischen Reife zu Außenseitern werden. Bleibt für aufmerksame KinderärztInnen die Aufgabe, Eltern von früh pubertierenden Mädchen zu mehr Aufmerksamkeit aufzufordern. 